\title{
The influence of maternal cortisol and emotional state during pregnancy on fetal intrauterine growth
}

Titia Hompes', Elske Vrieze', Steffen Fieuws², Annelies Simons³, Liesbeth Jaspers'1, Johan Van Bussel', Ganel Schops', Edith Gellens' ${ }^{1}$, Rieta Van Bree ${ }^{4}$, Johan Verhaeghe ${ }^{4}$, Bernard Spitz ${ }^{4}$, Koen Demyttenaere', Karel Allegaert ${ }^{5}$, Bea Van den Bergh ${ }^{3}$ and Stephan Claes $^{1}$

BACKGROUND: This exploratory study investigates the influence of maternal cortisol and emotional state during pregnancy on fetal intrauterine growth (IUG). We expected higher basal cortisol levels, or more depressive and anxious complaints during pregnancy, to be associated with slower IUG and lower birth weight.

METHODS: A total of 91 pregnant women were recruited from the antenatal clinic and were seen once each trimester. In addition to psychological assessments, a diurnal cortisol profile was derived from saliva samples. IUG was evaluated using ultrasound.

RESULTS: In mid-pregnancy (trimester (T)2), basal cortisol levels significantly predicted the variance of weight (proportion of variance in growth variable explained $(\mathrm{PVE})=11.6 \%)$ and body mass index (BMI) at birth (PVE $=6.8 \%$ ). In late pregnancy (T3) emotional state, particularly depressive symptoms (BMI at birth: $\mathrm{PVE}=6.9 \%$; ponderal index $(\mathrm{PI})$ at birth: $\mathrm{PVE}=8.2 \%$; head circumference at T3: PVE $=10.3 \%$; head circumference at birth PVE $=9.1 \%)$ and attachment (BMI at birth: PVE $=6.9 \%$; $\mathrm{PI}$ at birth: PVE $=7.2 \%$ ) had an influence on growth. Analysis of growth between T2 and T3 showed that attachment and cortisol in T3 had an influence on the variation in increase in estimated fetal weight (PVE $=12.5-8.6 \%$ ).

CONCLUSION: These data indicate basal cortisol levels were more important in T2 whereas emotional state was more important in T3.

$\mathbf{T}$ he research paradigm " $\mathrm{DOHaD}$ "-developmental origins of health and disease-encompasses the short- and longterm consequences of the prenatal and early postnatal environment for atypical as well as typical development later in life $(1,2)$.

In animal studies it has been shown that one of the key neurobiological mechanisms involved in the programming effects of prenatal stress, is the hypothalamic-pituitary-adrenal (HPA) axis of both mother and offspring. In humans, the mediating mechanisms in the transmission of stress from mother to fetus are still not clear, but here also the HPA axis is likely to be involved (3). There is good evidence for a strong correlation between cortisol in the maternal and fetal compartments $(4,5)$. Current data indicate that key targets for programming may include not only cortisol secretion itself, but also glucocorticoid receptor and $11 \beta$-hydroxysteroid dehydrogenase type 2 (11ßHSD2) gene expression in a range of tissues (6).

Eriksson (2) described birth size as a surrogate for summing the interaction between environmental and genetic influences in the prenatal period. In animal research, prenatal stress has been related to lower fetal and birth weight of the offspring $(7,8)$. In humans, results of research investigating the relationship between maternal distress and lower birth weight are inconsistent (9-14). Although there is evidence that maternal stress during pregnancy can lead to slower fetal growth, the variance explained by maternal stress is very low, i.e., about $1 \%$ in a meta-analysis of 35 studies (15).

It is unlikely that alterations in the function of the HPA axis are the only mechanism underlying low birth weight found after prenatal stress. In this respect, it is important to note that studies often show little correlation between various psychological measures and cortisol levels (3).

Given these data, we hypothesized that intrauterine growth (IUG) might be influenced by prenatal stress. We expected children from mothers with higher basal cortisol levels or with more depressive and anxious complaints during pregnancy to show slower IUG.

\section{RESULTS}

\section{Demographic Data}

A total of 91 pregnant women were included. Demographic data are summarized in Tables 1 and 2. The mean duration of pregnancy at inclusion was $10.8 \mathrm{wk}$, and $44 \%$ were primigravidae. The women had an average age of 30.0 (SD: 3.97; range: $22-37$ ) y. More than $70 \%$ of the mothers were highly educated; furthermore most of them were professionally active. Almost all mothers were married or living with a partner and were of the Belgian nationality. Children were born at an average of 39.05 postmenstrual weeks.

\footnotetext{
'Department of Psychiatry, University Hospitals Leuven, Leuven, Belgium; '2Leuven Biostatistics and Statistical Bioinformatics Centre (L-BioStat), Katholieke Universiteit Leuven, Leuven, Belgium; ${ }^{3}$ Department of Psychology, Tilburg University, Tilburg, The Netherlands; ${ }^{4}$ Department of Gynaecology and Obstetrics, University Hospitals Leuven, Leuven, Belgium; ${ }^{5}$ Department of Paediatrics, University Hospitals Leuven, Leuven, Belgium. Correspondence:Titia Hompes (titia.hompes@uz.kuleuven.be) 
Table 1. Demographic data (1)

\begin{tabular}{lcccc}
\hline & \multicolumn{4}{c}{ Weeks pregnant at examination during } \\
\cline { 2 - 5 } & T1 & T2 & T3 & Birth \\
\hline Min & 6 & 16 & 29 & 34 \\
Max & 14 & 27 & 39 & 41 \\
Mean & 10.78 & 23.61 & 34.90 & 39.05 \\
SD & 2.0 & 2.2 & 1.9 & 1.3 \\
\hline
\end{tabular}

Max, maximum; Min, minimum; T1, first trimester; $T 2$, second trimester;

T3, third trimester.

Table 2. Demographic data (2)

\begin{tabular}{llr}
\hline & Demographic data & $\%$ \\
\hline Education & Primary school & 2.2 \\
& Secondary school & 23.1 \\
& Higher education & 37.4 \\
& University & 35.2 \\
Unknown & 2.2 \\
Professional activity & Employee/laborer & 89.0 \\
& Independent & 4.4 \\
& Unemployed & 4.4 \\
& Staying at home & 2.2 \\
& Full time & 72.5 \\
Part time & 21.9 \\
Marital state & Not working & 6.6 \\
& Married/living together & 98.9 \\
Nationality & Single & 1.1 \\
& Belgian & 97.8 \\
& European & 1.1 \\
& Non-European & 1.1 \\
\hline
\end{tabular}

\section{Data on Fetal/Child Growth}

These data are summarized in Table 3 to give an overview on how growth was evaluated. We evaluated IUG through ultrasound as explained before.

\section{Data on Psychological Well-Being of the Mother}

These data are summarized in Table 4. Only a few mothers had a score equaling 10 or more on the Edinburgh Depression Scale (EDS), indicating that most mothers were not suffering from a major depressive illness. The prevalence in our study was lower than that stated by the Agency of Health Care Research and Quality (16): in T1: $10.7 \%$, in T2: 7.5\%, and in T3: 7.7\%. The scores on the Hospital Anxiety and Depression Scale (HADS) were mostly below the cut-off score of 8 for anxiety as well as for depression. On the HADS anxiety scale, $10.7 \%$ of the pregnant women had a score above 8 in T1, 16.4\% in T2, and $10.8 \%$ in T3. In the cohort study of Andersson (17), $11.4 \%$ of the included pregnant women were suffering from an anxiety disorder. The mean-score on the Pregnancy Related Anxiety Questionnaire (PRAQ) demonstrated medium levels of anxiety. The Maternal-Fetal Attachment Scale showed high attachment in each trimester.
Data on Basal Cortisol Day Profile and Area Under the Curve in Three Trimesters

As expected, we see a cortisol increase over the three trimesters (Table 5 and Figure 1). Between T1 and T3, cortisol levels were significantly different for all time points. A significant difference was seen between $\mathrm{T} 1$ and $\mathrm{T} 2$ at awakening and $30 \mathrm{~min}$ and $12 \mathrm{~h}$ later and between $\mathrm{T} 2$ and $\mathrm{T} 3$ at 4 and $12 \mathrm{~h}$ after awakening. Pregnancy is characterized by a progressive and significant increase in plasma concentrations of corticotropin-releasing hormone (18), adrenocorticotropic hormone, and cortisol $(19,20)$. Our result on cortisol awakening response and diurnal cortisol profile in pregnancy confirmed the results of other authors.

Depressive symptoms, anxiety symptoms, and attachment were not related to cortisol measurements. Therefore we can conclude that cortisol does not mediate the effects of these psychological measures on the obstetric outcomes. Where previous studies found a correlation between maternal emotional state and cortisol secretion, particularly in the third trimester $(5,21)$, this is not confirmed in our data.

\section{Principal Component Analysis and Regression Analysis}

In T1, the principal components (PCs) did not predict any of the growth parameters during gestation. The PC scores and cortisol in T2 (mid-pregnancy) (Table 6) explained 19.3\% ( $P=$ $0.016)$ of the variance in the weight at birth, $20.4 \%(P=0.003)$ of the variance in the body mass index (BMI) at birth, and $15.3 \%$ $(P=0.038)$ of the variance in the ponderal index $(\mathrm{PI})$ at birth in a multivariate setting. In this model, cortisol significantly predicted weight at birth (proportion of variance explained (PVE) $=11.6 \% ; P=0.006)$. In the multivariate model for prediction of BMI at birth, not only cortisol (PVE $=6.8 \%, P=0.016$ ), but also depression $(\mathrm{PVE}=10.7 \%, P=0.003)$ played a role, although the latter did not show a significant relation in a univariate setting $(P=0.245)$. All of these significant associations were negative. Although the high correlation between BMI at birth and PI at birth was strong (Pearson's $R: 0.890 ; P<0.001$ ), cortisol was not significantly associated with PI $(P=0.168)$, whereas depression was (PVE: $11.6 \%$; $P=0.006$ ).

In T3 (late pregnancy) (Table 7), we observed three interesting results. Again there was a tendency that some of the variance in BMI at birth (PVE: $14.0 \% ; P=0.063$ ), as well as the variance in the PI at birth (PVE: $14.8 \% ; P=0.101$ ), could be explained by the PCs and cortisol (area under the curve (AUC)). In addition to attachment (BMI at birth: $\mathrm{PVE}=6.9 \%, P=0.034 ; \mathrm{PI}$ at birth: $\mathrm{PVE}=7.2 \%, P=0.051)$, depression (BMI at birth: $\mathrm{PVE}=6.9 \%, P=0.035$; $\mathrm{PI}$ at birth: $\mathrm{PVE}=8.2 \%, P=0.038)$ was a significant component in the multivariate model. This relation was also present in the univariate model. Both depression and attachment in late pregnancy were negatively associated with BMI at birth and PI at birth.

Second, $24.0 \%(P=0.015)$ of the variation in head circumference (HC) in T3 was explained by the third-trimester PCs and cortisol (AUC) in a multivariate setting. Depression was the only significant component $(\mathrm{PVE}=9.0 \%, P=0.028)$ related 


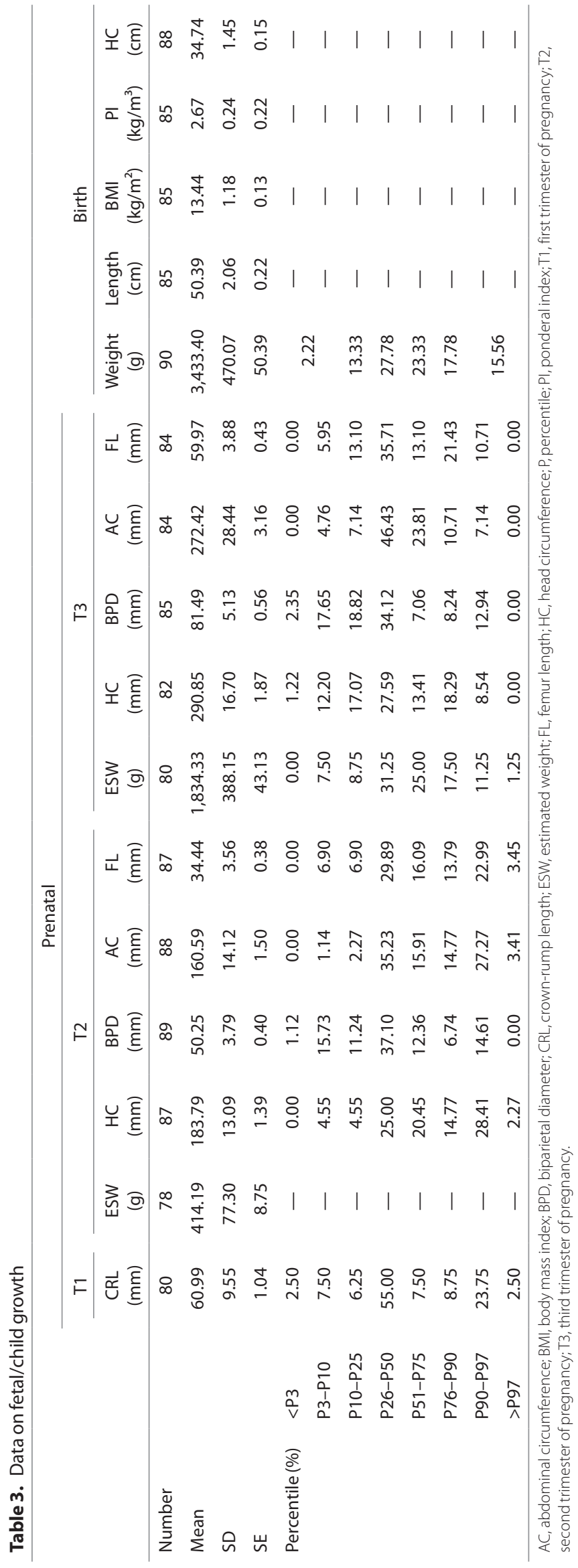

to the $\mathrm{HC}$ in $\mathrm{T} 3$, in the multivariate but also in the univariate setting ( $\mathrm{PVE}=10.3 \%, P=0.013)$. Cortisol was also significant in the multivariate model ( $\mathrm{PVE}=9.2 \%, P=0.026$ ). These associations were again in the negative direction.

Third, $12.1 \%(P=0.053)$ of the variation in $\mathrm{HC}$ at birth was explained by the third-trimester PCs and cortisol (AUC) in a multivariate setting. In a univariate setting anxiety $(\mathrm{PVE}=$ $9.5 \%, P=0.014)$ and depression $(\mathrm{PVE}=9.1 \%, P=0.015)$ were significant predictors, but this significance disappeared in the multivariate model.

In addition, we investigated the evolution of the different growth variables from the second to the third trimester (Table 8). The variance of the evolution of the biparietal diameter ((BPD) $\mathrm{PVE}=22.7 \%, P=0.022)$, the femur length $((\mathrm{FL}) \mathrm{PVE}=18.3 \%$, $P=0.043)$, and the abdominal circumference ((AC) PVE = $23.7 \%, P=0.017)$ are explained by the three PCs and cortisol. In the evolution of these growth variables again attachment plays an important role in both univariate (BPD: $\mathrm{PVE}=7.7 \%, P=0.032$; FL: $\mathrm{PVE}=9.8 \%, P=0.015 ; \mathrm{AC}: \mathrm{PVE}=9.8 \%, P=0.014)$ and multivariate (BPD: $\mathrm{PVE}=12.8 \%, P=0.010$; FL: $\mathrm{PVE}=13.2 \%$, $P=0.008$; AC: $\mathrm{PVE}=20.3 \%, P=0.001)$ models for all three variables. Cortisol showed a significant association in the multivariate models of the evolution of these three growth variables (BPD: PVE $=8.4 \%, P=0.035$; FL: PVE $=8.0 \%, P=0.035$; AC: $\mathrm{PVE}=8.1 \%, P=0.037$ ), although not in the univariate model. Anxiety has a role only in the univariate model of the evolution of BPD (PVE $=8.3 \%, P=0.025)$. All significant associations were negative.

Estimated weight (ESW) is calculated through the Hadlock formula, which contains BPD, AC, and FL. A total of $20.3 \%$ $(P=0.050)$ of the variation in increase in estimated fetal weight between the second and the third trimester was explained by the PCs and cortisol (AUC) in a multivariate setting. Given their influence in the models of the growth trajectories of $\mathrm{BPD}$, $\mathrm{AC}$, and FL, attachment (PVE $=12.5 \%, P=0.015)$ and cortisol $(\mathrm{PVE}=8.6 \%, P=0.042)$ were significant predictors in the multivariate model as expected. The latter was not significant in the univariate model $(P=0.196)$. These significant associations were again negative.

A total of $28.9 \%$ of the variance of difference in ratio of $\mathrm{HC} /$ AC between T2 and T3 is explained by the three PCs and cortisol $(P=0.003)$. In the multivariate model, attachment (PVE $=$ $7.0 \%, P=0.041)$ is important, whereas in the univariate model anxiety $(\mathrm{PVE}=12.5 \%, P=0.005)$ and depression $(\mathrm{PVE}=13.0 \%$, $P=0.004)$ are important. These are the only associations with which attachment is positively associated. All other significant associations are again negative. Attachment seems to be the only component that has a consistent influence on the evolution of the growth variables throughout the three trimesters.

\section{DISCUSSION}

This exploratory study shows evidence for the hypothesis that IUG is indeed influenced by prenatal maternal emotional state and/or maternal cortisol.

Our findings suggest that mid-pregnancy depressive symptoms are negatively associated with BMI and PI at birth, but no 
Table 4. Data on psychological well-being of the mother

\begin{tabular}{|c|c|c|c|c|c|c|c|c|c|c|c|c|c|c|}
\hline & & & \multicolumn{4}{|c|}{$\mathrm{T} 1$} & \multicolumn{4}{|c|}{$\mathrm{T} 2$} & \multicolumn{4}{|c|}{ T3 } \\
\hline & & & $N$ & Mean & SD & SE & $N$ & Mean & SD & SE & $N$ & Mean & SD & SE \\
\hline \multirow[t]{3}{*}{ EDPS } & & General & 84 & 6.46 & 4.47 & 0.49 & 67 & 6.05 & 4.09 & 0.50 & 65 & 6.12 & 4.08 & 0.51 \\
\hline & & Score $\geq 10$ & 17 & 13.12 & 3.69 & 0.89 & 12 & 12.67 & 2.57 & 0.74 & 10 & 13.40 & 3.84 & 1.21 \\
\hline & & Score $\geq 13$ & 9 & 15.00 & 4.24 & 1.41 & 5 & 15.00 & 2.35 & 1.05 & 6 & 15.33 & 3.88 & 1.58 \\
\hline \multirow[t]{4}{*}{ HADS } & Anxiety & General & 84 & 4.52 & 3.14 & 0.34 & 67 & 5.24 & 3.37 & 0.41 & 65 & 5.34 & 3.49 & 0.43 \\
\hline & & Score $>8$ & 9 & 11.11 & 2.57 & 0.86 & 11 & 11.17 & 2.12 & 0.64 & 7 & 13.06 & 3.74 & 1.42 \\
\hline & Depression & General & 84 & 3.58 & 3.50 & 0.38 & 67 & 3.29 & 2.63 & 0.32 & 65 & 3.80 & 2.53 & 0.31 \\
\hline & & Score $>8$ & 10 & 11.40 & 2.50 & 0.79 & 5 & 9.43 & 0.83 & 0.37 & 5 & 10.00 & 1.73 & 0.77 \\
\hline \multirow[t]{5}{*}{ PRAQ } & Fear of integrity & & & 3.63 & 1.40 & 0.15 & & 3.54 & 1.64 & 0.20 & & 3.42 & 1.61 & 0.20 \\
\hline & Fear of delivery & & & 3.10 & 1.52 & 0.16 & & 3.31 & 1.71 & 0.21 & & 3.74 & 1.65 & 0.21 \\
\hline & Fear of change & & & 2.60 & 1.32 & 0.14 & & 2.81 & 1.35 & 0.16 & & 2.81 & 1.30 & 0.16 \\
\hline & $\begin{array}{l}\text { Concern during } \\
\text { pregnancy }\end{array}$ & & 85 & 2.31 & 0.76 & 0.08 & 67 & 2.51 & 0.74 & 0.10 & 64 & 2.50 & 0.74 & 0.09 \\
\hline & $\begin{array}{l}\text { Concern about } \\
\text { future }\end{array}$ & & & 2.36 & 0.81 & 0.09 & & 2.56 & 0.96 & 0.12 & & 2.60 & 0.82 & 0.10 \\
\hline \multirow[t]{4}{*}{ MFAS } & $\begin{array}{l}\text { Anticipation on } \\
\text { interaction with } \\
\text { baby }\end{array}$ & & & 2.75 & 0.62 & 0.07 & & 3.01 & 0.63 & 0.08 & & 3.12 & 0.61 & 0.08 \\
\hline & Giving of self & & 85 & 3.41 & 0.49 & 0.05 & 67 & 3.32 & 0.48 & 0.06 & 64 & 3.41 & 0.43 & 0.05 \\
\hline & Name for baby & & & 2.38 & 1.11 & 0.12 & & 2.87 & 0.96 & 0.12 & & 3.21 & 0.77 & 0.10 \\
\hline & $\begin{array}{l}\text { Interaction with } \\
\text { fetus }\end{array}$ & & & 2.23 & 0.69 & 0.07 & & 2.68 & 0.59 & 0.07 & & 2.84 & 0.64 & 0.08 \\
\hline
\end{tabular}

EDPS, Edinburgh Depression Scale; HADS, Hospital Anxiety and Depression Scale; MFAS, Maternal and Fetal Attachment Scale; PRAQ, Pregnancy Related Anxiety Scale; T1, first trimester of pregnancy; 2 , second trimester of pregnancy; $T 3$, third trimester of pregnancy.

Table 5. Cortisol data

\begin{tabular}{|c|c|c|c|c|c|c|}
\hline \multirow[b]{2}{*}{ Trimester } & \multirow[b]{2}{*}{ Cortisol ( $\mu \mathrm{g} / \mathrm{dl})$} & \multicolumn{4}{|c|}{ Time (min) } & \multirow[t]{2}{*}{ AUC } \\
\hline & & 0 & 30 & 240 & 720 & \\
\hline First & Mean & 0.3719 & 0.4780 & 0.1895 & 0.0850 & 202.9282 \\
\hline \multirow[t]{2}{*}{ Second } & Mean & 0.4955 & 0.6098 & 0.2086 & 0.1155 & 241.8461 \\
\hline & SE & 0.0431 & 0.0453 & 0.0086 & 0.0098 & 16.1502 \\
\hline Third & SE & 0.0544 & 0.0565 & 0.0176 & 0.0292 & 23.2626 \\
\hline
\end{tabular}

Cortisol day profiles as well as area under the curve (AUC) in first, second, and third trimester; mean values at the four time points of sample taking as well as their SE are given.

correlation was found with birth weight. The Avon Longitudinal Study of Parents and Children (ALSPAC), however, reported an association with lower birth weight, although the effect was not statistically significant after adjustment for confounders, e.g., smoking (12). The small cross-sectional study of Diego et al. (22) showed an association between maternal psychological distress (anxiety, depression, and daily hassles) and fetal ESW in mid-pregnancy, whereas Henrichs et al. (23) described opposite findings. They adjusted for multiple confounders. The study of Henrichs et al. (23) was embedded in the Generation R Study, a population-based cohort study from fetal life onwards in Rotterdam, The Netherlands. The cohort includes 9,778 mothers and their children who were born between April 2002 and January 2006. Assessments in pregnant women consisted of physical examinations, fetal ultrasounds, biological samples, and questionnaires. One of the possible explanations for the discrepant findings between our study and the Generation R Study might be the different questionnaires used to assess depressive symptoms. Where Henrichs et al. (23) used the brief symptom inventory, we used the EDS and the HADS depression. However, given the fact that both ALSPAC and Generation R used large samples, it is more likely that these studies would more easily obtain statistically significant association. Furthermore, our study showed that mid-pregnancy cortisol secretion was negatively associated with weight and BMI at birth, although not with PI. More evidence for a correlation between fetal growth and cortisol secretion was found by Diego et al. (22) who reported a correlation between cortisol and ESW in mid-pregnancy. 


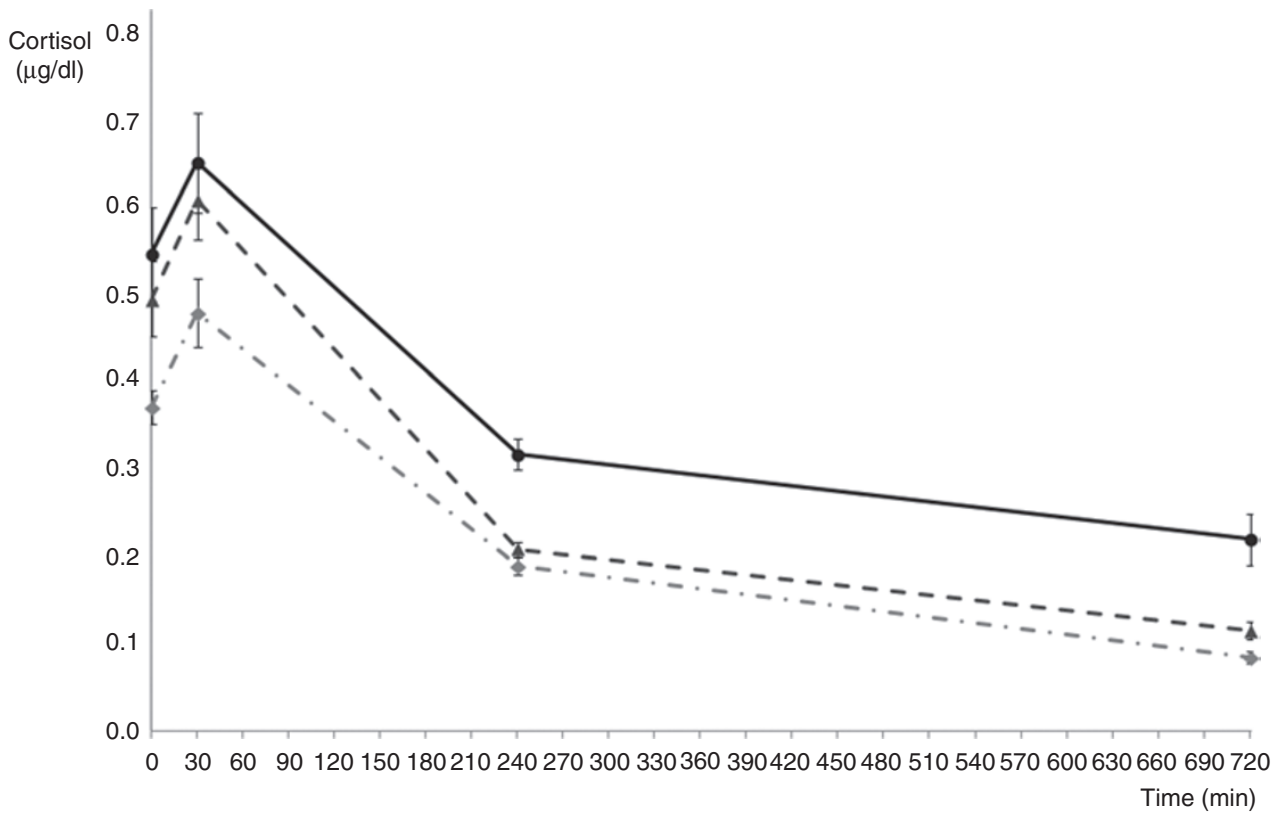

Figure 1. Basal cortisol day profiles. The light gray dashed line represents the first trimester, the dark gray dashed line represents the second trimester, and the black solid line represents the third trimester.

Table 6. Univariate and multivariate models mid-pregnancy

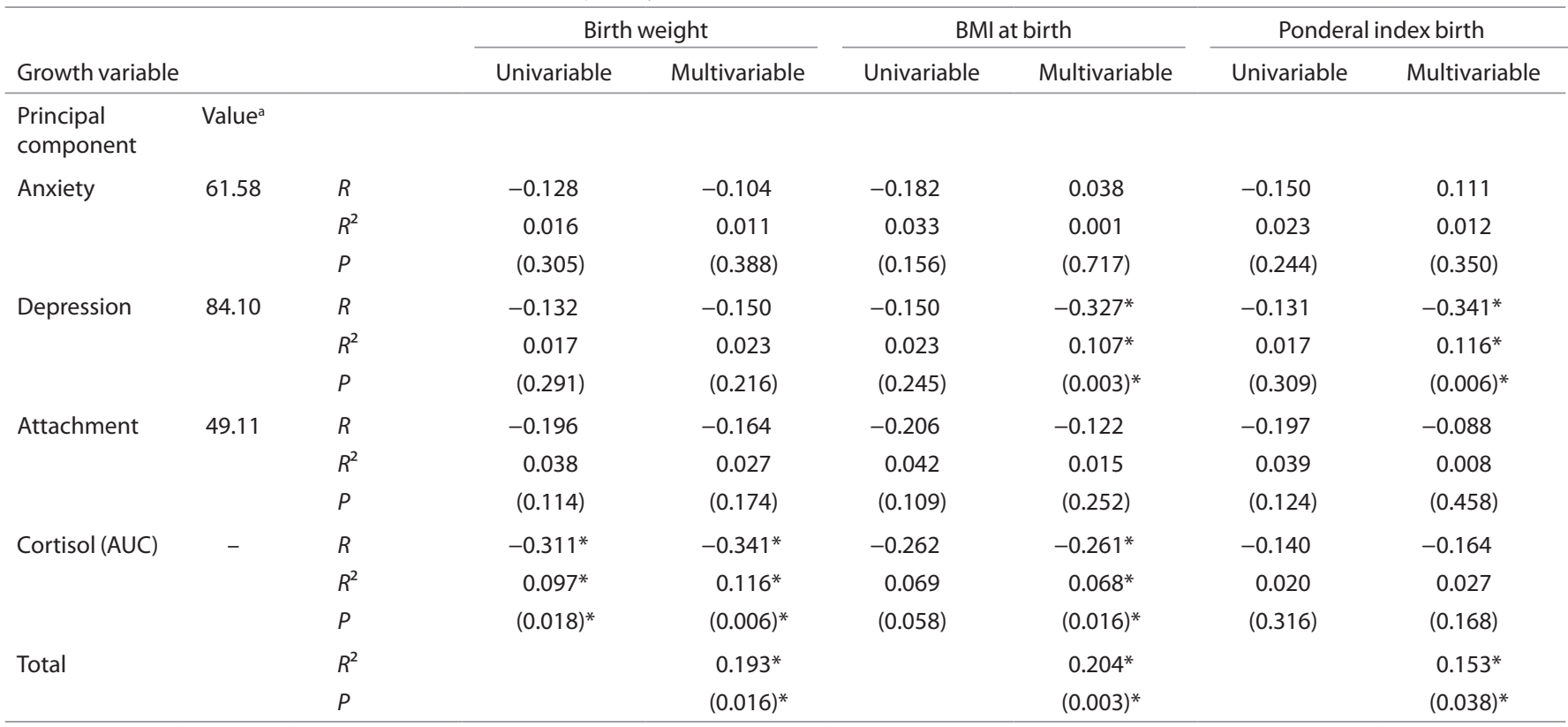

"Total" refers to the $R^{2}$ of all predictors in the multivariate model.

$A \cup C$, area under the curve; $R$, Pearson correlation (univariate) or semi-partial $R$ (multivariate); $R^{2}$, proportion of variance in growth variable explained (squared Pearson correlation in univariate setting and semi-partial $R^{2}$ in the multivariate model).

apercentage of variance in respective anxiety, depression, and attachment variables explained by the first principal component of the separate principal component analyses. *Significant values.

In late pregnancy, depressive symptoms were negatively associated with BMI and ponderal index at birth, but we did not find an effect of third-trimester cortisol on birth weight. Kivlighan et al. (24) reported steeper morning cortisol declines associated with lower infant birth weight. It is very difficult to compare because Kivlighan et al. applied other time points for salivary cortisol measurements. Where we used awakening as point of reference, the study of Kivlighan et al. used fixed time points $(8: 00 \mathrm{~h}, 12: 00 \mathrm{~h}$, and $16: 00 \mathrm{~h})$. In contrast to some published data $(9,23)$, anxiety did not specifically influence birth weight in our study. Explanations for this might be the different socio-economic background of our subjects as compared 
Table 7. Univariate and multivariate models: late pregnancy

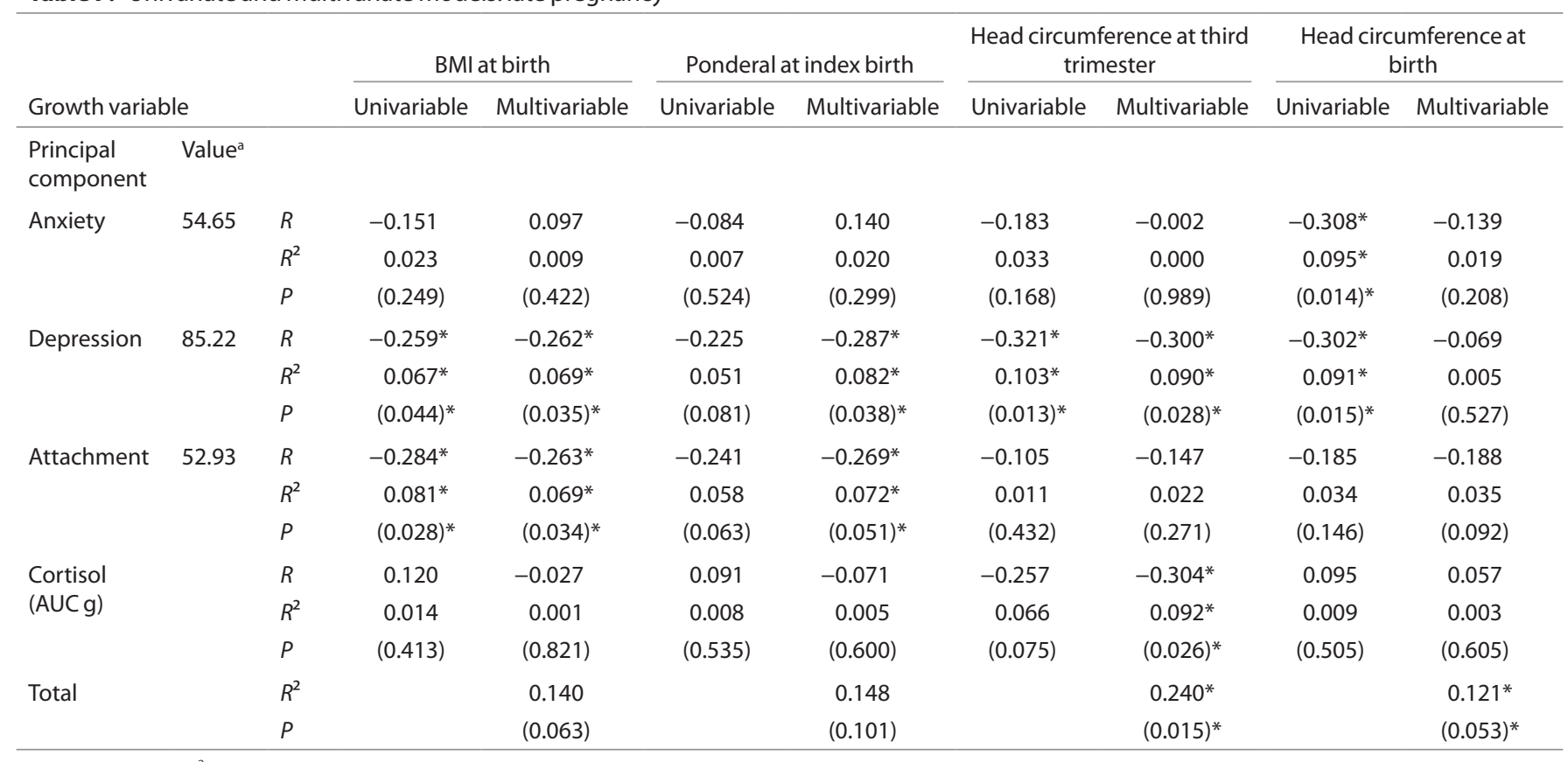

"Total" refers to the $R^{2}$ of all predictors in the multivariate model.

AUC, area under the curve; $R$, Pearson correlation (univariate) or semi-partial $R$ (multivariate); $R^{2}$, proportion of variance in growth variable explained (squared Pearson correlation in univariate setting and semi-partial $R^{2}$ in the multivariate model).

aPercentage of variance in respective anxiety, depression, and attachment variables explained by the first principal component of the separate principal component analyses. *Significant values.

with those of Rahman et al. (9) and the different questionnaires used to assess anxiety as compared with the Generation R Study (23).

In line with Henrichs et al. (23), we saw that depressive symptoms were negatively associated with HC. Fetal head growth can be seen as an indicator of fetal brain development, because HC correlates with brain volume (23). The ALSPAC study showed that HC and prenatal head growth was associated with subsequent IQ at the age of $4 \mathrm{y}$, although not at the age of 8 y (25). Henrichs et al. (23) hypothesized that fetal head growth might be a mediator in the relation of maternal prenatal psychological distress and subsequent child development.

Another important finding of our study is that attachment is a component that should be taken into account when studying prenatal stress. Attachment in late pregnancy was negatively associated with BMI at birth. Here we would have expected a positive association. It remains unclear what the explanation for this negative association might be. To our knowledge no studies have looked specifically at prenatal attachment and fetal growth.

To our knowledge, the study of Henrichs et al. (23) is the only one that provides insight on growth trajectories specifically between mid- and late pregnancy. We found a negative association between anxiety and the difference in ratio of $\mathrm{AC}$ and $\mathrm{HC}$ between mid- and late pregnancy. We did not find negative associations with the separate growth trajectories of the fetal head and abdomen that were seen in the study of Henrichs et al. (23). Depressive symptoms in late pregnancy were only associated with the difference in ratio of $\mathrm{AC}$ and $\mathrm{HC}$ between mid- and late pregnancy. Similar to the Generation R Study, our data did not show an association of depressive symptoms and femur and abdomen growth. We did not find the negative associations with fetal head growth and fetal weight gain that were reported by Henrichs et al. (23). Again, attachment seems to be the only component that has a consistent influence on the growth trajectories of the different growth variables throughout the three trimesters of pregnancy. It remains unclear to us why most of these associations are negative although we would expect them to be positive. Cortisol in late pregnancy seemed to have an influence on the growth trajectories of BPD, $\mathrm{AC}$, and FL. As a logical consequence, given that these three growth variables are important components of the Hadlock formula for ESW, cortisol in late pregnancy seems to influence the growth trajectory of ESW.

There are clear limitations to our study. As compared with the Generation R Study (23) and the ALSPAC study (12), our sample size is small. The protocol of our study is quite demanding for the mothers, which resulted in a dropout of more than $30 \%$. Furthermore, the women included here probably do not represent a random sample, as most of them were highly educated $(68.8 \%)$ and had a high socioeconomic status. Finally, the mean depression and anxiety scores were rather low. This, however, might also be an advantage, as the study shows the importance of depressive features and cortisol secretion during pregnancy in women without pronounced psychiatric symptoms. 


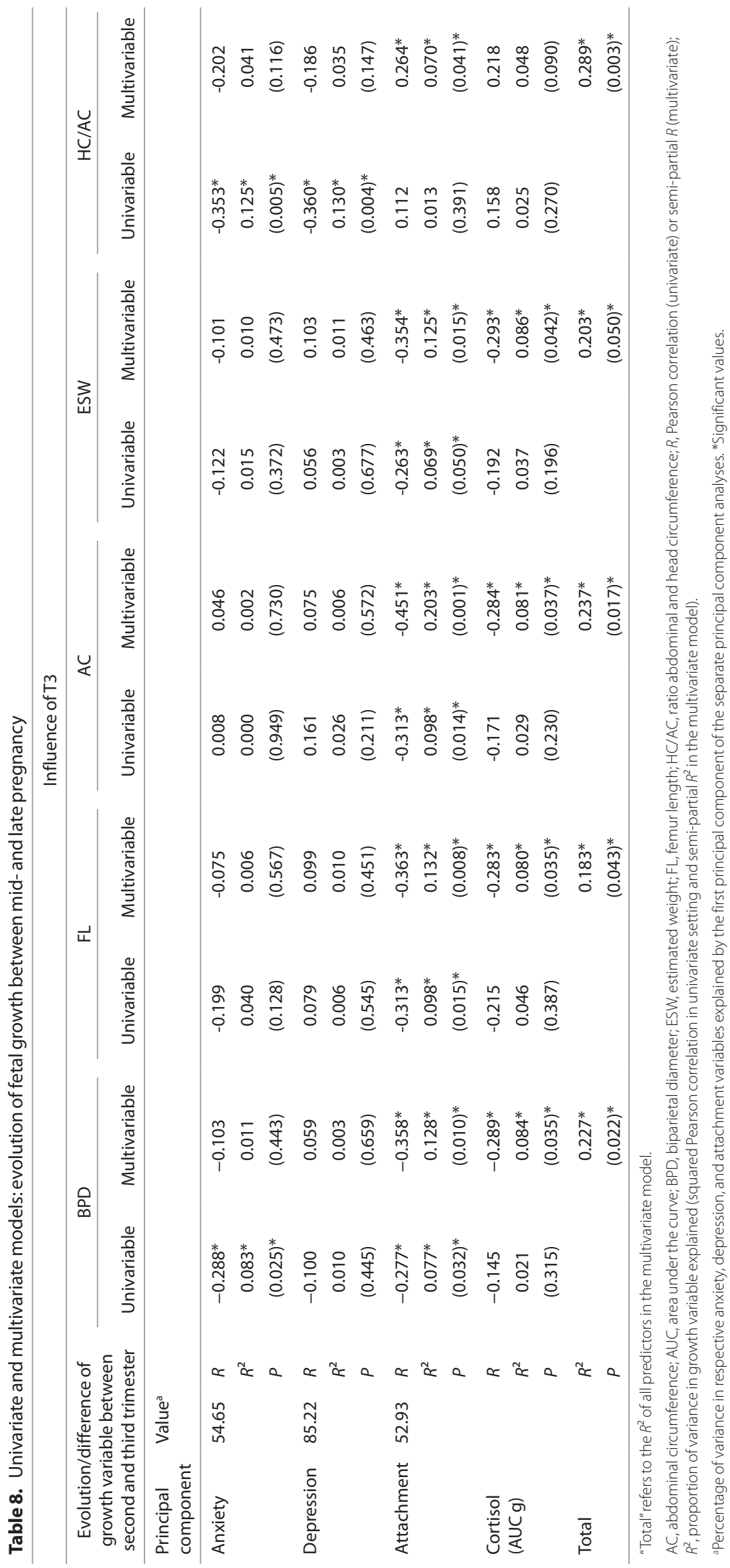


There are some important advantages to this study. First, we used ultrasonography to evaluate IUG. Until recently few studies have used ultrasound as a measure to evaluate the IUG of the fetus in relation to maternal distress during pregnancy. Birth outcomes like birth weight are used in several studies to investigate the influence of maternal prenatal distress, but these are only crude measures of IUG and cannot provide detailed and systematic information on the growth of the fetus across the different time periods in pregnancy (23). Another advantage is that in our study we did not only focus on the mid- and late pregnancy, but also on early pregnancy. Furthermore, we used cortisol day profiles, allowing a more detailed assessment of HPA axis function as compared with single cortisol samples. To our knowledge, there is no literature available on the effect of cortisol on growth during pregnancy as evaluated by ultrasonography.

This study shows preliminary evidence for the important role of maternal psychological factors and cortisol secretion on fetal development. Cortisol exerted an influence mainly in midpregnancy. We hypothesize that the fetus is more vulnerable to maternal cortisol in mid-pregnancy then in late pregnancy, because de novo cortisol production likely occurs transiently early in gestation (around 7-10 wk gestation). Due to the lack of expression of type $23 \beta$-hydroxysteroid dehydrogenase $/ \Delta^{4-5}$ isomerase, a crucial enzyme in the biosynthesis of cortisol, de novo cortisol biosynthesis appears to be suppressed until late gestation when cortisol production escalates. Mounting evidence indicates that cortisol may act as a "two-edged sword" for the fetus: it can promote maturation of fetal organs necessary for extra-uterine life, but it can also influence adversely fetal growth and postnatal development (26).

From our study, it can be concluded that depressive symptoms and attachment were particularly relevant during the third trimester and that, finally, attachment seems to have an influence on the growth trajectories of different growth variables between mid- and late pregnancy. These findings are important for preventive health care.

Evidently, these are exploratory data in a relatively small sample size. This study tried to find interesting patterns, although replication is needed, and further research concerning the underlying mechanisms and the effect of preventive measures should be performed.

\section{METHODS}

\section{Study Design}

We recruited pregnant women $(n=100)$ from the antenatal clinic of the University Hospitals in Leuven, Belgium, at about 8-12 wk gestation. Subsequently, they were examined once each trimester during pregnancy by our team. Seven pregnant women were excluded because they were suffering from somatic disorders or were taking corticosteroids or other medication inferring with the HPA axis. Multiple pregnancies $(n=2)$ were excluded because growth parameters in multiple pregnancies are not comparable with those of singleton pregnancies.

This study was approved by the ethical committee of the University Hospitals of Leuven, Belgium. Written informed consent was obtained from all participants.

\section{Clinical Assessment}

Table 9 shows the flow chart of this study, containing the general, psychiatric, and stress assessments of the mother and the assessments of the child.

General and medical information. Information was gathered on health, current professional activity, substance use, and a number of other relevant variables such as maternal weight before and weight gain during pregnancy. At birth, information on birth and well-being of mother and child, such as method of delivery; administration of epidural anesthesia; Apgar scores; height, weight, and $\mathrm{HC}$ of the baby; anatomy and weight of the placenta; complications during and after delivery; and other relevant variables were obtained from the medical file.

Fetal development and IUG. Fetal development and IUG were assessed through ultrasound examinations. Ultrasonography was conducted at set time points: around $12 \mathrm{wk}$ of gestational age, around $20 \mathrm{wk}$ gestational age, and around $30 \mathrm{wk}$ gestational age, by physicians and midwifes supervised by trained gynecologists.

Several variables were measured accurately using standardized techniques. Crown-rump length was obtained in the first trimester. In the second and the third trimester HC, BPD, AC and FL were measured. Furthermore, ESW was calculated using the formula by Hadlock (27) using $\mathrm{HC}$ and $\mathrm{AC}$ as well as FL, in the second and third trimester (before $18 \mathrm{wk}$ of gestation an accurate estimation of fetal weight cannot be achieved). The ratio of abdominal and HC, which is calculated by dividing AC by HC, measures symmetry of fetal growth (27). In addition to this the growth trajectories between T2 and T3 of the different growth variables (HC, BPD, AC, FL, and ESW) were examined. Therefore, we calculated the difference between the values measured in T2 and T3.

Table 9. Flowchart of the study

\begin{tabular}{|c|c|c|c|c|c|}
\hline & & \multicolumn{3}{|c|}{ During pregnancy } & At childbirth \\
\hline \multirow[t]{4}{*}{ Screening } & Mother & & & & \\
\hline & Questionnaires concerning emotional well-being & & & & \\
\hline & Edinburgh Depression Scale & + & + & + & \\
\hline & Pregnancy Related Anxiety Questionnaire & + & + & + & \\
\hline \multirow[t]{4}{*}{ Biological parameters } & Mother & & & & \\
\hline & Saliva: basal cortisol secretion $(4 \times / d-1 d)$ & + & + & + & \\
\hline & Child & & & & \\
\hline & Ultrasound evaluation & + & + & + & \\
\hline
\end{tabular}




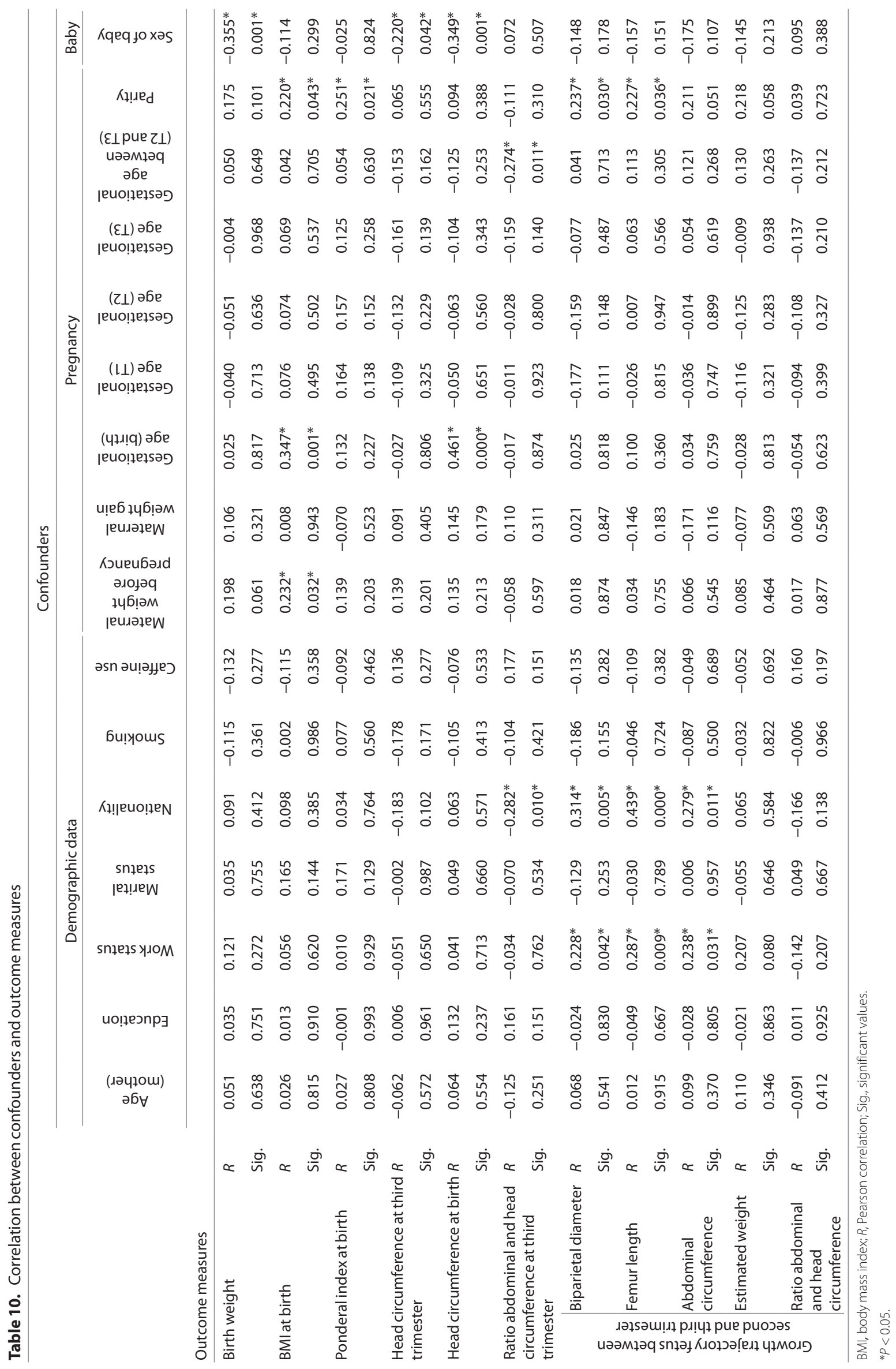


Other growth data (height, weight, and HC) were obtained from the medical file at birth. Percentiles were calculated using data from Flemish children $(28,29)$. The BMI (birth weight/length ${ }^{2}$ ) as well as the PI (birth weight/length ${ }^{3}$ ) was calculated.

Psychiatric and psychological assessments. Psychological well-being in pregnancy was assessed by means of a number of self-report, postal questionnaires each trimester. Prenatal depressive symptomatology was measured by the EDS (10 items with scores between 0 and 3 ) (30). Scores of 10 or more are worrisome. Scores of 13 or more may be an indication of a depressive state. In addition, the HADS (31) was assessed. The PRAQ (B.R. Van den Bergh, unpublished data) was used to measure specific fears and worries related to the participant's pregnancy. Simons and Van den Bergh (32) conducted a longitudinal study, in which pregnant women $(n=891)$, recruited in several university and general hospitals in Belgium, participated during T1 (8-14 wk), T2 (15-27 wk), and T3 (28-40 wk) of pregnancy. A simultaneous component analysis of the PRAQ revealed five subscales: fear for delivery (nine items; e.g., "I am afraid that I will lose a lot of blood during labor"), fear for the integrity of the baby (six items; e.g., "I am afraid that my baby will be brain damaged or lacking in mental capacity"), egocentric feelings/fear for changes (nine items; e.g., "I am concerned that my body will not regain its normal shape after the conclusion of pregnancy"), concerns about their own mood and the consequences for the baby ( 15 items; "I am concerned about my sudden mood changes"), concern about future mother-child, fatherchild, and partner relationship (16 items; "I am worried about my child rearing and parenting ability"). Responses are rated on a sevenpoint Likert scale ranging from "Does absolutely not apply" (1) to "Applies very well" (7). A high internal reliability for the total scale (Cronbach's $\alpha: \geq 0.95$ in T1, T2, and T3) as well as good internal reliability for each subscale (T1: $\alpha: \geq 0.77$; T2: $\alpha$ : $\geq 0.82$; and T3: $\alpha: \geq 0.73$ ) was found. Recently Van Bussel et al. (33) used the PRAQ in their study. They also found high internal reliabilities for the total scale ( 0.95 on T1, T2, and T3) and its subscales.

We decided on using multiple measures for anxiety and depression. Regarding depressive symptoms we used the EDS and the HADS depression, which are scales that both measure depression although they question different aspects of depression and in this way they are complementary to each other. As to anxiety symptoms, we used a specific pregnancy-related questionnaire, PRAQ, and the HADS anxiety, which is a more generalized measure of anxiety. Again both questionnaires highlight different aspects of anxiety and therefore are complementary. By using both questionnaires we get a broader and more accurate view on the anxiety symptoms present.

The mother-fetus relationship was measured by the Maternal-Fetal Attachment Scale (34), containing 17 items with scores from 1-4. Simultaneous component analysis revealed four subscales: (i) anticipation of interaction with the baby (e.g., I talk to my unborn baby), (ii) interaction with the fetus (e.g., I picture myself feeding the baby), (iii) giving of self (e.g., I give up doing certain things because I want to help my baby), and (iv) choice of name (e.g., I have decided on a name for a girl baby) (34). For the Dutch version, factor analysis revealed four subscales with the following three having a good internal consistency (i.e., $\alpha>0.70$ ) "anticipation on interaction with the baby", "interaction with the fetus", "giving of self" (35).

HPA axis activity assessment. Mothers collected saliva samples for cortisol once during each trimester at four different time points: at awakening, and $30 \mathrm{~min}$, and 4 and $12 \mathrm{~h}$ later by using Sorbette (Salimetrics, Suffolk, UK). Mothers were asked to note the exact time point of sample taking. Detailed instructions with photographs were provided. The collected samples were stored in Eppendorf tubes in the refrigerator until returning them in prepaid and addressed envelopes. On arrival at the lab they were frozen at $-20^{\circ} \mathrm{C}$ until centrifugation. After being thawed, the samples were centrifuged at 3,000 rpm for $15 \mathrm{~min}$. To determine cortisol levels in saliva a High Sensitivity Salivary Cortisol Enzyme Immunoassay Kit (Salimetrics) was used. This assay was designed to capture the full range of salivary cortisol levels $(0.003-3.0 \mu \mathrm{g} / \mathrm{dl})$ while using only $25 \mu \mathrm{l}$ of saliva per test and is resilient to the effects of interference caused by collection techniques that affect $\mathrm{pH}$. All four samples were used to calculate the AUC using the trapezoidal rule. The exact time in minutes between two samples was taken into account.

\section{Statistical Analysis}

Statistical analysis was performed using SPSS 18.0. In each trimester univariate and multivariate regression models were used to explore the relation between each specific growth variable, as well as the growth trajectory between second and third trimesters of the different growth variables (HC, BPD, AC, FL, and ESW), on the one hand and the psychological assessments and cortisol data on the other hand. To reduce the number of predictors in the models, a principal component analysis was performed on each of the following groups of variables, for each pregnancy trimester: anxiety variables (HADS anxiety and PRAQ subscales), depression variables (EDS and HADS depression) and attachment variables (MFAS). The subject's scores on the first PCs, summarizing these three domains, were used as predictors in the models. Cortisol (AUC) was used as a fourth predictor. In the regression model we used the growth variables as described before or, when available, the percentiles resulting from these growth variables as dependent variables. Where possible, percentiles were used as these take into account the exact gestational or postnatal age of the baby.

Gestational age at birth, sex of the baby, maternal weight before and weight gain during pregnancy, maternal age, smoking during pregnancy, parity, and education were examined as potential confounders of the outcome measures. Because of the large number of confounders only those showing significant correlations with the outcome variable $(P<0.05)$ in the univariate setting were included in the multivariate model. No model reduction strategies were considered for the predictors of interest. $P$ values smaller than 0.05 are considered significant (Table 10). Because of the exploratory character of the study, no corrections for multiple testing have been made. Therefore, a single significant $P$ value should be interpreted carefully.

Variance inflation factors in all models were maximally two, hence no important multicolinearity was present.

\section{STATEMENT OF FINANCIAL SUPPORT}

K.A. and S.C. are supported by the Fund for Scientific Research, Flanders (Belgium) (F.W.O. Vlaanderen) through a Fundamental Clinical Investigatorship (grants $1800209 \mathrm{~N}$ and $1800411 \mathrm{~N}$ ).

\section{REFERENCES}

1. Wadhwa PD, Buss C, Entringer S, Swanson JM. Developmental origins of health and disease: brief history of the approach and current focus on epigenetic mechanisms. Semin Reprod Med 2009;27:358-68.

2. Eriksson J. Patterns of growth: relevance to developmental origins of health and disease. In: Gluckman PD, Hanson MA, eds. Developmental Origins of Health and Disease. Cambridge, UK: Cambridge University Press, 2006:223-232.

3. Glover V, O'Connor TG, O'Donnell K. Prenatal stress and the programming of the HPA axis. Neurosci Biobehav Rev 2010;35:17-22.

4. Gitau R, Fisk NM, Teixeira JM, Cameron A, Glover V. Fetal hypothalamic-pituitary-adrenal stress responses to invasive procedures are independent of maternal responses. J Clin Endocrinol Metab 2001;86:104-9.

5. Sarkar P, Bergman K, Fisk NM, Glover V. Maternal anxiety at amniocentesis and plasma cortisol. Prenat Diagn 2006;26:505-9.

6. Bertram CE, Hanson MA. Prenatal programming of postnatal endocrine responses by glucocorticoids. Reproduction 2002;124:459-67.

7. Pinto ML, Shetty PS. Influence of exercise-induced maternal stress on fetal outcome in Wistar rats: inter-generational effects. Br J Nutr 1995;73: 645-53.

8. Lesage J, Del-Favero F, Leonhardt M, et al. Prenatal stress induces intrauterine growth restriction and programmes glucose intolerance and feeding behaviour disturbances in the aged rat. J Endocrinol 2004;181:291-6.

9. Rahman A, Bunn J, Lovel H, Creed F. Association between antenatal depression and low birthweight in a developing country. Acta Psychiatr Scand 2007;115:481-6. 
10. Nordentoft M, Lou HC, Hansen D, et al. Intrauterine growth retardation and premature delivery: the influence of maternal smoking and psychosocial factors. Am J Public Health 1996;86:347-54.

11. Andersson L, Sundström-Poromaa I, Wulff M, Aström M, Bixo M. Neonatal outcome following maternal antenatal depression and anxiety: a population-based study. Am J Epidemiol 2004;159:872-81.

12. Evans J, Heron J, Patel RR, Wiles N. Depressive symptoms during pregnancy and low birth weight at term: longitudinal study. Br J Psychiatry 2007;191:84-5.

13. Bhagwanani SG, Seagraves K, Dierker LJ, Lax M. Relationship between prenatal anxiety and perinatal outcome in nulliparous women: a prospective study. J Natl Med Assoc 1997;89:93-8.

14. McDonald RL. The role of emotional factors in obstetric complications: a review. Psychosom Med 1968;30:222-43.

15. Littleton HL, Bye K, Buck K, Amacker A. Psychosocial stress during pregnancy and perinatal outcomes: a meta-analytic review. J Psychosom Obstet Gynaecol 2010;31:219-28.

16. Gaynes BN, Gavin N, Meltzer-Brody S, et al. Perinatal depression: prevalence, screening accuracy, and screening outcomes. Evid Rep Technol Assess (Summ) 2005;119:1-8.

17. Andersson L, Sundström-Poromaa I, Wulff M, Aström M, Bixo M. Depression and anxiety during pregnancy and six months postpartum: a followup study. Acta Obstet Gynecol Scand 2006;85:937-44.

18. Schulte HM, Weisner D, Allolio B. The corticotrophin releasing hormone test in late pregnancy: lack of adrenocorticotrophin and cortisol response. Clin Endocrinol (Oxf) 1990;33:99-106.

19. de Weerth C, Buitelaar JK. Cortisol awakening response in pregnant women. Psychoneuroendocrinology 2005;30:902-7.

20. Levine A, Zagoory-Sharon O, Feldman R, Lewis JG, Weller A. Measuring cortisol in human psychobiological studies. Physiol Behav 2007;90:43-53.

21. Obel C, Hedegaard M, Henriksen TB, Secher NJ, Olsen J, Levine S. Stress and salivary cortisol during pregnancy. Psychoneuroendocrinology 2005;30:647-56.

22. Diego MA, Jones NA, Field T, et al. Maternal psychological distress, prenatal cortisol, and fetal weight. Psychosom Med 2006;68:747-53.

23. Henrichs J, Schenk JJ, Roza SJ, et al. Maternal psychological distress and fetal growth trajectories: the Generation R Study. Psychol Med 2010;40:633-43.
24. Kivlighan KT, DiPietro JA, Costigan KA, Laudenslager ML. Diurnal rhythm of cortisol during late pregnancy: associations with maternal psychological well-being and fetal growth. Psychoneuroendocrinology 2008;33:1225-35.

25. Gale CR, O'Callaghan FJ, Bredow M, Martyn CN; Avon Longitudinal Study of Parents and Children Study Team. The influence of head growth in fetal life, infancy, and childhood on intelligence at the ages of 4 and 8 years. Pediatrics 2006;118:1486-92.

26. Ishimoto $\mathrm{H}$, Jaffe RB. Development and function of the human fetal adrenal cortex: a key component in the feto-placental unit. Endocr Rev 2011:32:317-55.

27. Hadlock FP, Harrist RB, Carpenter RJ, Deter RL, Park SK. Sonographic estimation of fetal weight. The value of femur length in addition to head and abdomen measurements. Radiology 1984;150:535-40.

28. Devlieger H, Martens G, Bekaert A, Eeckels R. Standards for birthweight adjusted for gestational age in the flemish newborn. Tijdschr voor geneeskunde 2000;56:1-14.

29. Laboratorium voor antropogenetica Vrije Universiteit Brussel. Flemish growth charts 2004. (http://www.vub.ac.be/groeicurven/english.html.) Accessed 4 July 2006.

30. Cox JL, Holden JM, Sagovsky R. Detection of postnatal depression. Development of the 10-item Edinburgh Postnatal Depression Scale. Br J Psychiatry 1987;150:782-6.

31. Zigmond AS, Snaith RP. The hospital anxiety and depression scale. Acta Psychiatr Scand 1983;67:361-70.

32. Simons A, Van den Bergh BR. Maternal fears in pregnancy. Poster presentation "Conference of Emotions" 22-24 October 2007, Tilburg, The Netherlands.

33. van Bussel JC, Spitz B, Demyttenaere K. Anxiety in pregnant and postpartum women. An exploratory study of the role of maternal orientations. J Affect Disord 2009;114:232-42.

34. Cranley MS. Development of a tool for the measurement of maternal attachment during pregnancy. Nurs Res 1981;30:281-4.

35. Michiels L, Van den Eynden K. Angst tijdens de zwangerschap? Conceptuele beschrijving en interventiestrategieën. [Anxiety during pregnancy: a specific phenomenon? Conceptual description and intervention strategies.] Dissertation to obtain a degree of license in psychology. Leuven, Belgium, 2006. 